\title{
CONSISTENCY AND ASYMPTOTIC NORMALITY OF STOCHASTIC EULER SCHEMES FOR ORDINARY DIFFERENTIAL EQUATIONS
}

\author{
JOHANNES T. N. KREBS
}

\begin{abstract}
General stochastic Euler schemes for ordinary differential equations are studied. We give proofs on the consistency, the rate of convergence and the asymptotic normality of these procedures.
\end{abstract}

\section{INTRODUCTION}

We study the consistency and asymptotic normality of stochastic Euler schemes which are designed to approximate ordinary differential equations. Euler schemes are often used to simulate stochastic differential equations. Fierro and Torres [2001] study the consistency of these schemes in the context of Itô stochastic differential equations. However, this idea can be used to approximate ordinary differential equations, too: Fierro and Torres [2007] consider a special kind of Euler approximation for a given ODE. In this paper, we generalize the idea: Let there be given the ODE system $\dot{x}=F(t, x), x(0)=x_{0} \in \mathbb{R}^{d}, t \in[0, T], 0<T<$ $\infty$. Then we approximate the solution $x$ on a partition $\pi^{N}$ of $[0, T]$ with a stochastic Euler scheme that is based on random variables $\tilde{F}_{k}^{N}$ instead on $F$. This approach can be useful in applications where one aims at approximating the trajectory of such a solution $x$ for a function $F$ which is costly to evaluate, for instance, in the case where $F$ is the sum of (finitely) many single functions $f_{i}, i \in I$, i.e. $F=\sum_{i \in I} f_{i}$. The paper is organized as follows: In Section 2 we introduce the basic notions and regularity conditions of the model. In Section 3 we give consistency results for our general Euler scheme. We state results on the asymptotic normality of the procedure in Section 4 Appendix $\mathrm{A}$ contains some background material.

\section{Preliminaries}

We denote for $p \geq 1$ by $\|\cdot\|_{p}$ the $p$-norms on the $d$-dimensional Euclidean space. Let $T \in \mathbb{R}_{+}$be a finite time horizon and let $F=\left(F_{1}, \ldots, F_{d}\right)^{\prime}:[0, T] \times \mathbb{R}^{d} \rightarrow \mathbb{R}^{d}$ be a continuous vector valued function. $F$ fulfills the following growth conditions w.r.t. the first and second coordinate for $s, t \in[0, T]$ and for $x, y \in \mathbb{R}^{d}$

$$
\begin{aligned}
& \|F(s, x)-F(t, x)\|_{1} \leq K_{1}\left(1+\|x\|_{1}\right)|s-t|, \\
& \|F(t, x)-F(t, y)\|_{1} \leq K_{2}\|x-y\|_{1},
\end{aligned}
$$

where $0<K_{1}, K_{2}<\infty$ are some positive constants. Let there be given the ODE $\dot{x}=F(t, x)$ and $x(0)=x_{0} \in \mathbb{R}^{d}$ on $[0, T]$. Denote the unique global solution of this equation by $x:[0, T] \rightarrow \mathbb{R}^{d}, x(t):=x(0)+\int_{0}^{t} F(s, x(s)) \mathrm{d} s$. This solution is guaranteed by the global Lipschitz condition (2.2) and is Lipschitz-continuous with a Lipschitzconstant $0<C<\infty$, i.e. $\|x(s)-x(t)\|_{1} \leq C|s-t|$. Next, choose a sequence of partitions, $\pi^{N}, N \in \mathbb{N}_{+}$, of the interval $[0, T]$ such that $\pi^{N}$ consists of the points $t_{0}^{N}=0<t_{1}^{N}<\ldots<t_{K_{N}}^{N}=T$ and such that the mesh of the partition $\Delta^{N}:=\max _{1 \leq k \leq K_{N}} \Delta_{k}^{N}$ converges to zero as $N \rightarrow \infty$, where $\Delta_{k}^{N}:=t_{k}^{N}-t_{k-1}^{N}$. The stochastic part is introduced via a probability space $(\Omega, \mathcal{A}, \mathbb{P})$ endowed with the following mappings: For $N \in \mathbb{N}_{+}$and $k=1, \ldots, K_{N}$ the function

$$
\tilde{F}_{k}^{N}=\left(\tilde{F}_{k, 1}^{N}, \ldots, \tilde{F}_{k, d}^{N}\right)^{\prime}:[0, T] \times \mathbb{R}^{d} \times \Omega \rightarrow \mathbb{R}^{d} \text { is measurable }\left[\mathcal{B}\left([0, T] \times \mathbb{R}^{d}\right) \otimes \mathcal{A} \mid \mathcal{B}\left(\mathbb{R}^{d}\right)\right]
$$

and is Lipschitz-continuous w.r.t. the second coordinate with the same Lipschitz constant as $F$.

Furthermore, for any selection of time-space coordinates $\left(t_{1}, y_{1}\right), \ldots,\left(t_{K_{N}}, y_{K_{N}}\right) \in[0, T] \times \mathbb{R}^{d}$ the random variables $\tilde{F}_{1}^{N}\left(t_{1}, y_{1}\right), \ldots, \tilde{F}_{K_{N}}^{N}\left(t_{K_{N}}, y_{K_{N}}\right)$ are independent and each $\tilde{F}_{k}^{N}$ is an unbiased estimator of $F$ in the sense that $\mathbb{E}\left[\tilde{F}_{k}^{N}(t, y)\right]=F(t, y)$ for $(t, y) \in[0, T] \times \mathbb{R}^{d}$. In addition, we assume that there exists a constant

Date: December, 12015.

2010 Mathematics Subject Classification. Primary: 60H10; secondary 34F05, 93E03.

Key words and phrases. Stochastic Euler schemes; ordinary differential equations; consistency; rate of convergence; asymptotic normality.

The author gratefully acknowledges the financial support of the Fraunhofer ITWM which is part of the Fraunhofer-Gesellschaft zur Förderung der angewandten Forschung e.V. 
$0<K_{3}<\infty$ such that for each $t \in[0, T], a=1, \ldots, d, k=1, \ldots, K_{N}$ and $N \in \mathbb{N}_{+}$the variance of the approximation is bounded as $\operatorname{Var}\left[\tilde{F}_{k, a}^{N}(t, x(t))\right] \leq K_{3}$. We generate for each $N \in \mathbb{N}_{+}$a stochastic sequence $\hat{x}^{N}=\left\{\hat{x}^{N}\left(t_{i}^{N}\right): i=0, \ldots, K_{N}\right\}$ according to the rule

$$
\hat{x}^{N}\left(t_{0}^{N}\right):=x(0) \in \mathbb{R}^{d} \text { and } \hat{x}^{N}\left(t_{i}^{N}\right):=\hat{x}^{N}\left(t_{i-1}^{N}\right)+\Delta_{i}^{N} \tilde{F}_{i}^{N}\left(t_{i-1}^{N}, \hat{x}^{N}\left(t_{i-1}^{N}\right)\right) \text { for } i=1, \ldots, K_{N} .
$$

We pass from this sequence $\left\{\hat{x}^{N}\left(t_{i}^{N}\right): i=0, \ldots, K_{N}\right\}$ to a right-continuous process which we denote again by $\hat{x}^{N}$, namely, we define

$$
\hat{x}^{N}(t):=\hat{x}^{N}\left(t_{i}^{N}\right) \text { for } t \in\left[t_{i}^{N}, t_{i+1}^{N}\right) \text { for } i=0, \ldots, K_{N}-1 \text { and } \hat{x}^{N}(T)=\hat{x}^{N}\left(t_{K_{N}}^{N}\right) .
$$

In the following, when speaking of $\hat{x}^{N}$, we shall always refer to this càdlàg process. Moreover, $\left\{\mathcal{F}^{N}(\cdot): N \in \mathbb{N}_{+}\right\}$ is a sequence of filtrations on $(\Omega, \mathcal{A}, \mathbb{P})$ such that for each $N \in \mathbb{N}_{+}$the filtration $\mathcal{F}^{N}(\cdot)$ is the natural and rightcontinuous filtration of the process $\hat{x}^{N}$ from equation (2.4).

\section{Consistency and Rate of Convergence}

We come to the first main result of this paper, this is the convergence in mean of the processes $\hat{x}^{N}, N \in \mathbb{N}_{+}$, namely

Theorem 3.1 ( $L^{2}$-convergence of $\hat{x}^{N}$ to $\left.x\right)$. Let the sequence of stochastic processes $\left(\hat{x}^{N}: N \in \mathbb{N}_{+}\right)$be defined in equations (2.3) and (2.4). Let $x$ be the unique global solution to the ordinary differential equation. Then, there exists a constant $0<B<\infty$ such that

$$
\left\|\sup _{t \in[0, T]}\right\| \hat{x}^{N}(t)-x(t)\left\|_{1}\right\|_{L^{2}(\mathbb{P})} \leq B \sqrt{\Delta^{N}}
$$

Proof. Throughout the proof we shall write $\|\cdot\|$ for the Euclidean 1-norm on $\mathbb{R}^{d}$. Furthermore, we set $x_{k}^{N}:=$ $x^{N}\left(t_{k}^{N}\right)$ and $\hat{x}_{k}^{N}:=\hat{x}^{N}\left(t_{k}^{N}\right)$, for $k=0, \ldots, K_{N}$. First, we consider $\hat{x}^{N}$ at the points $t_{k}^{N}, k=1, \ldots, K_{n}$. We derive for the difference $\hat{x}_{k}^{N}-x_{k}^{N}$ at each $k=1, \ldots, K_{N}$ the equation

$$
\begin{aligned}
\hat{x}_{k}^{N}-x_{k}^{N}= & \hat{x}_{k-1}^{N}-x_{k-1}^{N}+\left\{\tilde{F}_{k}^{N}\left(t_{k-1}^{N}, \hat{x}_{k-1}^{N}\right)-\tilde{F}_{k}^{N}\left(t_{k-1}^{N}, x_{k-1}^{N}\right)\right\} \Delta_{k}^{N}+\left\{\tilde{F}_{k}^{N}\left(t_{k-1}^{N}, x_{k-1}^{N}\right)-F\left(t_{k-1}^{N}, x_{k-1}^{N}\right)\right\} \Delta_{k}^{N} \\
& +\left\{F\left(t_{k-1}^{N}, x_{k-1}^{N}\right) \Delta_{k}^{N}-\int_{\Delta_{k}^{N}} F(s, x(s)) \mathrm{d} s\right\} .
\end{aligned}
$$

Successive iteration down to $t_{0}^{N}$ yields

$$
\begin{aligned}
\hat{x}_{k}^{N}-x_{k}^{N}= & \sum_{j=1}^{k}\left\{\tilde{F}_{j}^{N}\left(t_{j-1}^{N}, \hat{x}_{j-1}^{N}\right)-\tilde{F}_{j}^{N}\left(t_{j-1}^{N}, x_{j-1}^{N}\right)\right\} \Delta_{j}^{N}+\sum_{j=1}^{k}\left\{\tilde{F}_{j}^{N}\left(t_{j-1}^{N}, x_{j-1}^{N}\right)-F\left(t_{j-1}^{N}, x_{j-1}^{N}\right)\right\} \Delta_{j}^{N} \\
& +\sum_{j=1}^{k}\left\{F\left(t_{j-1}^{N}, x_{j-1}^{N}\right) \Delta_{j}^{N}-\int_{\Delta_{j}^{N}} F(s, x(s)) \mathrm{d} s\right\} .
\end{aligned}
$$

By the growth condition w.r.t. the time coordinate and the Lipschitz condition w.r.t. the space coordinate, we have for the last term in 3.1)

$$
\left\|\int_{\Delta_{j}^{N}} F\left(t_{j-1}^{N}, x_{j-1}^{N}\right)-F(s, x(s)) \mathrm{d} s\right\| \leq\left\{K_{1}\left(1+\sup _{t \in[0, T]}\|x(t)\|\right)+K_{2} C\right\}\left(\Delta_{j}^{N}\right)^{2} .
$$

We put for short $L:=K_{1}\left(1+\sup _{t \in[0, T]}\|x(t)\|\right)+K_{2} C$. We can estimate the left-hand side of (3.1) using the Lipschitz condition on the stochastic approximations $\tilde{F}_{k}^{N}$ of $F$ to arrive at the following bound for $\left\|\hat{x}_{k}^{N}-x_{k}^{N}\right\|$ :

$$
\left\|\hat{x}_{k}^{N}-x_{k}^{N}\right\| \leq K_{2} \sum_{j=1}^{k}\left\|\hat{x}_{j-1}^{N}-x_{j-1}^{N}\right\| \Delta_{j}^{N}+\left\|\sum_{j=1}^{k}\left(\tilde{F}_{j}^{N}\left(t_{j-1}^{N}, x_{j-1}^{N}\right)-F\left(t_{j-1}^{N}, x_{j-1}^{N}\right)\right) \Delta_{j}^{N}\right\|+\sum_{j=1}^{k} L\left(\Delta_{j}^{N}\right)^{2} .
$$

We apply the discrete Gronwall inequality from LemmaA.1 from Appendix A to the bound given in (3.2). We get

$$
\left\|\hat{x}_{k}^{N}-x_{k}^{N}\right\| \leq\left\|\sum_{j=1}^{k}\left(\tilde{F}_{j}^{N}\left(t_{j-1}^{N}, x_{j-1}\right)-F\left(t_{j-1}^{N}, x_{j-1}^{N}\right)\right) \Delta_{j}^{N}\right\|+\sum_{j=1}^{k} L\left(\Delta_{j}^{N}\right)^{2}
$$


CONSISTENCY AND ASYMPTOTIC NORMALITY OF STOCHASTIC EULER SCHEMES FOR ORDINARY DIFFERENTIAL EQUATIONS 3

$$
\begin{aligned}
& +\sum_{j=1}^{k-1}\left\{\left\|\sum_{i=1}^{j}\left(\tilde{F}_{i}^{N}\left(t_{i-1}^{N}, x_{i-1}^{N}\right)-F\left(t_{i-1}^{N}, x_{i-1}^{N}\right)\right) \Delta_{i}^{N}\right\|+L \sum_{i=1}^{j}\left(\Delta_{i}^{N}\right)^{2}\right\}\left\{K_{2} \Delta_{j}^{N}\right\} \exp \left(\sum_{i=j+1}^{k-1} K_{2} \Delta_{i}^{N}\right) \\
\leq & M_{1} \Delta^{N}+\left\|\sum_{j=1}^{k}\left(\tilde{F}_{j}^{N}\left(t_{j-1}^{N}, x_{j-1}^{N}\right)-F\left(t_{j-1}^{N}, x_{j-1}^{N}\right)\right) \Delta_{j}^{N}\right\| \\
& +M_{2}\left\{\sum_{j=1}^{k-1}\left\|\sum_{i=1}^{j}\left(\tilde{F}_{i}^{N}\left(t_{i-1}^{N}, x_{i-1}^{N}\right)-F\left(t_{i-1}^{N}, x_{i-1}^{N}\right)\right) \Delta_{i}^{N}\right\| \Delta_{j}^{N}\right\},
\end{aligned}
$$

where the constants $M_{1}$ and $M_{2}$ are given by $M_{1}:=L T+K_{2} L T^{2} \exp \left(K_{2} T\right)$ and $M_{2}:=K_{2} \exp \left(K_{2} T\right)$. In particular, for $A:=2 M_{1}^{2}$ and $B:=2\left(1+M_{2} T\right)^{2}$ it holds good that

$$
\sup _{1 \leq j \leq K_{N}}\left\|\hat{x}_{j}^{N}-x_{j}^{N}\right\|^{2} \leq A\left(\Delta^{N}\right)^{2}+B \sup _{1 \leq j \leq K_{N}}\left\|\sum_{i=1}^{j}\left(\tilde{F}_{i}^{N}\left(t_{i-1}^{N}, x_{i-1}^{N}\right)-F\left(t_{i-1}^{N}, x_{i-1}^{N}\right)\right) \Delta_{i}^{N}\right\| \|^{2} .
$$

Next, we use the independence assumptions on the $\tilde{F}_{k}^{N}$ and the assumption that in each point $\mathbb{E}\left[\tilde{F}_{k}^{N}(t, x)\right]=$ $F(t, x)$. We show that the discrete process

$$
\left\{t_{0}^{N}, \ldots, t_{K_{N}}^{N}\right\} \ni t_{j}^{N} \mapsto\left\|\sum_{i=1}^{K_{n}} 1_{\left\{t_{i}^{N} \leq t_{j}^{N}\right\}}\left(\tilde{F}_{i}^{N}\left(t_{i-1}^{N}, x\left(t_{i-1}^{N}\right)\right)-F\left(t_{i-1}^{N}, x\left(t_{i-1}^{N}\right)\right)\right) \Delta_{i}^{N}\right\|
$$

constitutes a submartingale $\left[\mathcal{F}^{N}(\cdot)\right]$. Indeed, we have for any two $1 \leq j \leq k \leq K_{N}$

$$
\mathbb{E}\left[\left\|\sum_{i=1}^{k}\left(\tilde{F}_{i}^{N}\left(t_{i-1}^{N}, x_{i-1}^{N}\right)-F\left(t_{i-1}^{N}, x_{i-1}^{N}\right)\right) \Delta_{i}^{N}\right\| \mid \mathcal{F}^{N}\left(t_{j}^{N}\right)\right]=\mathbb{E}\left[\sum_{a=1}^{d}\left|\sum_{i=1}^{k}\left(\tilde{F}_{i, a}^{N}\left(t_{i-1}^{N}, x_{i-1}^{N}\right)-F_{a}\left(t_{i-1}^{N}, x_{i-1}^{N}\right)\right) \Delta_{i}^{N}\right| \mid \mathcal{F}^{N}\left(t_{j}^{N}\right)\right] .
$$

Due to the independence assumption on the stochastic family $\tilde{F}_{i}^{N}$, the 1-dimensional processes

$$
\left\{t_{0}^{N}, \ldots, t_{K_{N}}^{N}\right\} \ni t_{j}^{N} \mapsto\left|\sum_{i=1}^{K_{N}} 1_{\left\{t_{i}^{N} \leq t_{j}^{N}\right\}}\left(\tilde{F}_{i, a}^{N}\left(t_{i-1}^{N}, x\left(t_{i-1}^{N}\right)\right)-F_{a}\left(t_{i-1}^{N}, x\left(t_{i-1}^{N}\right)\right)\right) \Delta_{i}^{N}\right|
$$

are submartingales $\left[\mathcal{F}^{N}(\cdot)\right]$ for each $a=1, \ldots, d$. Summation over the index $a$ proves the statement about the submartingale property. This puts us in position to use Doob's $L^{p}$-Inequality for equation (3.4) with $p=2$ applied to the above submartingale

$$
\begin{aligned}
\mathbb{E}\left[\sup _{1 \leq k \leq K_{N}}\left\|\hat{x}_{k}^{N}-x_{k}^{N}\right\|^{2}\right] & \leq A\left(\Delta^{N}\right)^{2}+B \mathbb{E}\left[\sup _{1 \leq k \leq K_{N}}\left(\left\|\sum_{i=1}^{k}\left(\tilde{F}_{i}^{N}\left(t_{i-1}^{N}, x_{i-1}^{N}\right)-F\left(t_{i-1}^{N}, x_{i-1}^{N}\right)\right) \Delta_{i}^{N}\right\|\right)^{2}\right] \\
& \leq A\left(\Delta^{N}\right)^{2}+4 B \mathbb{E}\left[\left(\left\|\sum_{i=1}^{K_{N}}\left(\tilde{F}_{i}^{N}\left(t_{i-1}^{N}, x_{i-1}^{N}\right)-F\left(t_{i-1}^{N}, x_{i-1}^{N}\right)\right) \Delta_{i}^{N}\right\|\right)^{2}\right] \\
& \leq A\left(\Delta^{N}\right)^{2}+4 B d \sum_{a=1}^{d} \mathbb{E}\left[\left|\sum_{i=1}^{K_{N}}\left(\tilde{F}_{i, a}^{N}\left(t_{i-1}^{N}, x_{i-1}^{N}\right)-F_{a}\left(t_{i-1}^{N}, x_{i-1}^{N}\right)\right) \Delta_{i}^{N}\right|^{2}\right] \\
& =A\left(\Delta^{N}\right)^{2}+4 B d \sum_{a=1}^{d} \sum_{i=1}^{K_{N}}\left(\Delta_{i}^{N}\right)^{2} \operatorname{Var}\left[\tilde{F}_{i, a}^{N}\left(t_{i-1}^{N}, x_{i-1}^{N}\right)\right] \\
& \leq A\left(\Delta^{N}\right)^{2}+4 B T d^{2} K_{3} \Delta^{N} .
\end{aligned}
$$

The first inequality (3.5) follows immediately from inequality (3.4). Inequality (3.6) stems from Doob's $L^{p}$ inequality. Equality (3.7) follows from the indepence of the random variables $\tilde{F}_{1, a}^{N}\left(t_{0}^{N}, x\left(t_{0}^{N}\right)\right), \ldots, \tilde{F}_{K_{N}, a}^{N}\left(t_{K_{N}-1}^{N}, x\left(t_{K_{N}-1}^{N}\right)\right)$. The last inequality (3.8) follows from the condition that the variance of the approximation is uniformly bounded. We are now in position to consider the processes $\hat{x}^{N}$ over the entire interval $[0, T]$. Remember that $\hat{x}^{N}(t)=\hat{x}^{N}\left(t_{k-1}^{N}\right)$ for $t \in\left[t_{k-1}^{N}, t_{k}^{N}\right)$ and $\hat{x}^{N}(T)=\hat{x}^{N}\left(t_{N}^{N}\right)$, thus,

$$
\sup _{t \in[0, T]}\left\|\hat{x}^{N}(t)-x(t)\right\|^{2} \leq 2\left\{\sup _{1 \leq k \leq K_{N}}\left\|\hat{x}^{N}\left(t_{k}^{N}\right)-x\left(t_{k}^{N}\right)\right\|^{2}+C^{2}\left(\Delta^{N}\right)^{2}\right\} .
$$


All in all, we find that $\left\|\sup _{t \in[0, T]}\right\| \hat{x}^{N}(t)-x(t)\|\|_{L^{2}(\mathbb{P})} \leq$ const $\left(\Delta^{N}\right)^{\frac{1}{2}}$ for a sequence of partitions having a mesh $\Delta^{N}$ which converges to zero. This finishes the proof.

In addition to the $L^{2}(\mathbb{P})$-convergence of the process $\hat{x}^{N}$, we can state another result on the pathwise convergence for a special choice of the partitioning sequence $\left\{\pi^{N}: N=1, \ldots, \infty\right\}$. It is an application of Kolmogorov's maximal inequality and follows immediately from the inequality from equation (3.4). We have the following theorem

Theorem 3.2 (a.s.-convergence of $\hat{x}^{N}$ ). Let $\left\{\pi^{N}: N=1, \ldots, \infty\right\}$ be a partitioning sequence of the interval $[0, T]$ such that $\sum_{N=1}^{\infty} \Delta^{N}<\infty$. Then $\sup _{t \in[0, T]}\left\|\hat{x}^{N}(t)-x(t)\right\|_{1}$ converges to zero almost surely.

Proof. Write again $x_{k}^{N}:=x\left(t_{k}^{N}\right)$ and $\hat{x}_{k}^{N}:=\hat{x}^{N}\left(t_{k}^{N}\right)$ for $k=0, \ldots, K_{N}$. Consider equation (3.4), the maximum on the right-hand side can be bounded as

$$
\max _{1 \leq j \leq K_{N}}\left\|\sum_{i=1}^{j}\left(\tilde{F}_{i}^{N}\left(t_{i-1}^{N}, x_{i-1}^{N}\right)-F\left(t_{i-1}^{N}, x_{i-1}^{N}\right)\right) \Delta_{i}^{N}\right\|_{1} \leq \sum_{a=1}^{d}\left(\max _{1 \leq j \leq K_{N}}\left|\sum_{i=1}^{j}\left(\tilde{F}_{i, a}^{N}\left(t_{i-1}^{N}, x_{i-1}^{N}\right)-F_{a}\left(t_{i-1}^{N}, x_{i-1}^{N}\right)\right) \Delta_{i}^{N}\right|\right) .
$$

We show that $\max _{1 \leq j \leq K_{N}}\left|\sum_{i=1}^{j}\left(\tilde{F}_{i, a}^{N}\left(t_{i-1}^{N}, x_{i-1}^{N}\right)-F_{a}\left(t_{i-1}^{N}, x_{i-1}^{N}\right)\right) \Delta_{i}^{N}\right| \rightarrow 0$ almost surely for each coordinate $a=1, \ldots, d$. An application of Kolmogorov's maximal inequality yields for $\varepsilon>0$ that

$$
\mathbb{P}\left(\max _{1 \leq j \leq K_{N}}\left|\sum_{i=1}^{j}\left(\tilde{F}_{i, a}^{N}\left(t_{i-1}^{N}, x_{i-1}^{N}\right)-F_{a}\left(t_{i-1}^{N}, x_{i-1}^{N}\right)\right) \Delta_{i}^{N}\right|>\varepsilon\right) \leq \frac{1}{\varepsilon^{2}} \sum_{i=1}^{K_{N}} \operatorname{Var}\left(\tilde{F}_{i, a}^{N}\left(t_{i-1}^{N}, x_{i-1}^{N}\right) \Delta_{i}^{N}\right) \leq \frac{K_{3} T}{\varepsilon^{2}} \Delta^{N} .
$$

Hence, we conclude the $a . s$--convergence from the first Borel-Cantelli Lemma by the convergence assumption on the meshes of partitioning sequence $\left\{\pi^{N}: N=1, \ldots, \infty\right\}$. The conclusion follows immediately by combining inequality (3.4) and (3.9), as well as the fact that almost sure convergence is unaffected by continuous transformations.

\section{Asymptotic Normality of Stochastic Approximation Procedures}

In this section we prove the asymptotic normality of the stochastic Euler schemes for ODE approximations

Theorem 4.1. Let $\left\{\pi^{N}: N \in \mathbb{N}_{+}\right\}$be the sequence of dyadic partitions of $[0, T]$, i.e. $\pi^{N}=\left\{T k / 2^{N}: k=\right.$ $\left.0,1, \ldots, 2^{N}\right\}$. Let $F=\left(F_{1}, \ldots, F_{d}\right)$ fulfill the regularity conditions from (2.1) and 2.2). Additionally, let each component of $F$ be continuously differentiable w.r.t. the space coordinate, i.e. $(t, x) \mapsto \nabla_{x} F_{i}(t, x)$ is continuous for $i=1, \ldots, d$.

Furthermore, let the stochastic approximations $\tilde{F}_{k}^{N}$ be regular in that for all $N \in \mathbb{N}_{+}$and $k=1, \ldots, 2^{N}$ the $\tilde{F}_{k}^{N}$ are independent copies of $\tilde{F}$ where the time-space process $\tilde{F}:[0, T] \times \mathbb{R}^{d} \times \Omega \rightarrow \mathbb{R}^{d}$ is Lipschitz-continuous in the space coordinate with the Lipschitz constant $K_{2}$ as well as continuous in the time coordinate and fulfills the integrability condition

$$
\mathbb{E}\left[\sup _{t \in[0, T]}\left\|\tilde{F}(t, x(t)) \cdot \tilde{F}(t, x(t))^{\prime}\right\|_{1}^{2}\right]<\infty
$$

Then for each $t \in[0, T]$ in the limit $\lim _{N \rightarrow \infty}\left(\Delta^{N}\right)^{-\frac{1}{2}}\left(\hat{x}^{N}(t)-x(t)\right) \sim \mathcal{N}(0, \Sigma(t))$, where the function $\Sigma:[0, T] \rightarrow$ $\mathbb{R}^{d \times d}$ is defined as

$$
\Sigma(t)=\int_{0}^{t} P(s, t) \mathbb{E}\left[(\tilde{F}(s, x(s))-F(s, x(s))) \cdot(\tilde{F}(s, x(s))-F(s, x(s)))^{\prime}\right] P(s, t)^{\prime} \mathrm{d} s
$$

and $P$ is the uniform limit of the function $P^{N}$ on $[0, T]^{2}$ given by $[0, T]^{2} \ni P^{N}(s, t)=\prod_{s<t_{j}^{N} \leq t}\left(I+\Delta^{N} \nabla_{x} F\left(t_{j-1}^{N}, x\left(t_{j-1}^{N}\right)\right)\right)$.

Proof. We write $\|\cdot\|$ throughout the proof for the 2-norm; since any two norms on the Euclidean space are equivalent, bounds and estimates w.r.t. the 1-norm can be multiplied with the corresponding equivalence constant and are thus valid w.r.t. the 2-norm, too. For a matrix $A$, denote by $\|A\|:=\sup _{x:\|x\| \leq 1}\|A x\|$ the spectral norm of $A$. We use the abbreviations

$$
Z^{N}:=\left(\Delta^{N}\right)^{-\frac{1}{2}}\left(\hat{x}^{N}-x\right) \text { as well as } x_{k}^{N}:=x\left(t_{k}^{N}\right) \text { and } \hat{x}_{k}^{N}:=\hat{x}^{N}\left(t_{k}^{N}\right)
$$


for simplicity. Choose $t \in[0, T]$ arbitrary but fix, w.l.o.g. $t \in\left[t_{k}^{N}, t_{k+1}^{N}\right)$, if we add the virtual point $t_{2^{N}+1}^{N}$ in case that $t=T$. Then

$$
\begin{aligned}
\hat{x}^{N}(t)-x(t)= & \hat{x}_{k}^{N}-x_{k}^{N}+\left(x_{k}^{N}-x(t)\right)=\hat{x}_{k-1}^{N}-x_{k-1}^{N}+\Delta^{N} \tilde{F}_{k}^{N}\left(t_{k-1}^{N}, \hat{x}_{k-1}^{N}\right)-\int_{t_{k-1}^{N}}^{t_{k}^{N}} F(s, x(s)) \mathrm{d} s-\int_{t_{k}^{N}}^{t} F(s, x(s)) \mathrm{d} s \\
= & {\left[I+\Delta^{N} \nabla_{x} F\left(t_{k-1}^{N}, x_{k-1}^{N}\right)\right]\left(\hat{x}_{k-1}^{N}-x_{k-1}^{N}\right)+\Delta^{N}\left[\tilde{F}_{k}^{N}\left(t_{k-1}^{N}, x_{k-1}^{N}\right)-F\left(t_{k-1}^{N}, x_{k-1}^{N}\right)\right] } \\
& +\Delta^{N}\left[\tilde{F}_{k}^{N}\left(t_{k-1}^{N}, \hat{x}_{k-1}^{N}\right)-F\left(t_{k-1}^{N}, \hat{x}_{k-1}^{N}\right)-\left(\tilde{F}_{k}^{N}\left(t_{k-1}^{N}, x_{k-1}^{N}\right)-F\left(t_{k-1}^{N}, x_{k-1}^{N}\right)\right)\right] \\
& +\Delta^{N}\left[F\left(t_{k-1}^{N}, \hat{x}_{k-1}^{N}\right)-F\left(t_{k-1}^{N}, x_{k-1}^{N}\right)-\nabla_{x} F\left(t_{k-1}^{N}, x_{k-1}^{N}\right)\left(\hat{x}_{k-1}^{N}-x_{k-1}^{N}\right)\right] \\
& +\int_{t_{k-1}^{N}}^{t_{k}^{N}} F\left(t_{k-1}^{N}, x_{k-1}^{N}\right)-F(s, x(s)) \mathrm{d} s-\int_{t_{k}^{N}}^{t} F(s, x(s)) \mathrm{d} s
\end{aligned}
$$

We make the following definitions

$$
\begin{aligned}
& m_{k}^{N}:=\tilde{F}_{k}^{N}\left(t_{k-1}^{N}, x_{k-1}^{N}\right)-F\left(t_{k-1}^{N}, x_{k-1}^{N}\right), \\
& R_{1}^{N, k}:=\Delta^{N}\left[\tilde{F}_{k}^{N}\left(t_{k-1}^{N}, \hat{x}_{k-1}^{N}\right)-\tilde{F}_{k}^{N}\left(t_{k-1}^{N}, x_{k-1}^{N}\right)-\left(F\left(t_{k-1}^{N}, \hat{x}_{k-1}^{N}\right)-F\left(t_{k-1}^{N}, x_{k-1}^{N}\right)\right)\right], \\
& R_{2}^{N, k}:=\Delta^{N}\left[\left(F\left(t_{k-1}^{N}, \hat{x}_{k-1}^{N}\right)-F\left(t_{k-1}^{N}, x_{k-1}^{N}\right)\right)-\nabla_{x} F\left(t_{k-1}^{N}, x_{k-1}^{N}\right)\left(\hat{x}_{k-1}^{N}-x_{k-1}^{N}\right)\right], \\
& R_{3}^{N, k}:=\int_{t_{k-1}^{N}}^{t_{k}^{N}} F\left(t_{k-1}^{N}, x_{k-1}^{N}\right)-F(s, x(s)) \mathrm{d} s \text { and } R_{4}^{N}:=-\int_{t_{k}^{N}}^{t} F(s, x(s)) \mathrm{d} s .
\end{aligned}
$$

Set $R^{N, k}:=R_{1}^{N, k}+R_{2}^{N, k}+R_{3}^{N, k}$; note that $\left|R_{4}^{N}\right| \leq C \Delta^{N}$, for a constant $0<C<\infty$. Thus, we get $Z^{N}(t)=$ $Z^{N}\left(t_{k}^{N}\right)+\left(\Delta^{N}\right)^{-\frac{1}{2}} R_{4}^{N}$ and at the partitioning points, we face the following structure

$$
Z^{N}\left(t_{k}^{N}\right)=\left[I+\Delta^{N} \nabla_{x} F\left(t_{k-1}^{N}, x_{k-1}^{N}\right)\right] Z^{N}\left(t_{k-1}^{N}\right)+\left(\Delta^{N}\right)^{\frac{1}{2}} m_{k}^{N}+\left(\Delta^{N}\right)^{-\frac{1}{2}} R^{N, k} \text { for } 0<t_{k}^{N} \leq T \text { and } Z^{N}(0)=0 .
$$

Consequently, successive iteration yields

$$
Z^{N}(t)=\sum_{k: t_{0}^{N}<t_{k}^{N} \leq t}\left\{\prod_{j: t_{k}^{N}<t_{j}^{N} \leq t}\left(I+\Delta_{j}^{N} \nabla_{x} F\left(t_{j-1}^{N}, x_{j-1}^{N}\right)\right)\right\}\left\{\left(\Delta^{N}\right)^{\frac{1}{2}} m_{k}^{N}+\left(\Delta^{N}\right)^{-\frac{1}{2}} R^{N, k}\right\}+R_{4}^{N} .
$$

In the sequel, we prove that the sum which involves the $m_{k}^{N}$ tends to the desired normal distribution, whereas the sum involving the remainder $R^{N, k}$ tends to zero in probability. Hence, $Z^{N}(t)$ is asymptotically normally distributed with the same parameters. Consider the first sum, we use the definitions

$$
U_{N, k}:=\prod_{j: t_{k}^{N}<t_{j}^{N} \leq t}\left\{I+\Delta_{j}^{N} \nabla_{x} F\left(t_{j-1}^{N}, x_{j-1}^{N}\right)\right\}\left(\Delta^{N}\right)^{\frac{1}{2}} m_{k}^{N} \text { for } 0<t_{k}^{N} \leq t
$$

and $U_{N}:=\sum_{0<t_{k}^{N} \leq t} U_{N, k}$. Note that for $N \in \mathbb{N}_{+}$the random variables $U_{N, 1}, \ldots, U_{N, 2^{N}}$ are independent. W.l.o.g., assume that $(\Omega, \mathcal{A}, \mathbb{P})$ is endowed with independent normal distributions $Y_{N, k}$ such that $Y_{N, k} \sim$ $\mathcal{N}\left(0, \operatorname{Cov}\left[U_{N, k}, U_{N, k}\right]\right)$ for $k=1, \ldots, 2^{N}$. Set $Y_{N}:=\sum_{0<t_{k}^{N} \leq t} Y_{N, k}$. We prove that the difference of the characteristic functions $\varphi_{U_{N}}-\varphi_{Y_{N}}$ convergences pointwise to zero: For a fix $\alpha \in \mathbb{R}^{d}$, we show that $\varphi_{U_{N}}(\alpha)-\varphi_{Y_{N}}(\alpha) \rightarrow 0$ as $N \rightarrow \infty$. Therefore, we use the fundamental inequality

$$
\left\|\varphi_{U_{N}}(\alpha)-\varphi_{Y_{N}}(\alpha)\right\|_{\mathbb{C}} \leq \sum_{k: 0<t_{k}^{N} \leq t}\left\|\varphi_{U_{N, k}}(\alpha)-\varphi_{Y_{N, k}}(\alpha)\right\|_{\mathbb{C}} .
$$

An application of Lemma A.2 yields

$$
\begin{aligned}
\text { (4.2) } \leq & 2\|\alpha\|^{2} \sum_{k: 0<t_{k}^{N} \leq t} \mathbb{E}\left[\left\|U_{N, k}\right\|^{2} 1_{\left\{\|\alpha\|\|\| U_{N, k} \|>\varepsilon / 2\right\}}\right]+\varepsilon\|\alpha\|^{2} \sum_{k: 0<t_{k}^{N} \leq t} \mathbb{E}\left[\left\|U_{N, k}\right\|^{2}\right] \\
& +2\|\alpha\|^{2} \sum_{k: 0<t_{k}^{N} \leq t} \mathbb{E}\left[\left\|Y_{N, k}\right\|^{2} 1_{\left\{\|\alpha\|\left\|Y_{N, k}\right\|>\varepsilon / 2\right\}}\right]+\varepsilon\|\alpha\|^{2} \sum_{k: 0<t_{k}^{N} \leq t} \mathbb{E}\left[\left\|Y_{N, k}\right\|^{2}\right]
\end{aligned}
$$

We show that the first and the third sum of (A.2) converge to zero as $N \rightarrow \infty$ for any $\varepsilon>0$. This implies that the second and the fourth sum are bounded, and, when multiplied by $\varepsilon$, become small, too. We intend to 
bound $\left\|U_{N, k}\right\|^{2}$, it is

$$
\left\|U_{N, k}\right\| \leq\left(\Delta^{N}\right)^{\frac{1}{2}} \prod_{j: t_{k}^{N}<t_{j}^{N} \leq t}\left(1+\Delta^{N}\left\|\nabla_{x} F(\cdot, x(\cdot))\right\|_{\infty}\right)\left\|m_{k}^{N}\right\| \leq\left(\Delta^{N}\right)^{\frac{1}{2}} \exp \left(T\left\|\nabla_{x} F(\cdot, x(\cdot))\right\|_{\infty}\right)\left\|m_{k}^{N}\right\| .
$$

We consider the first sum of (4.3): Using (4.4), we arrive at

$$
\begin{aligned}
\sum_{k: 0<t_{k}^{N} \leq t} \mathbb{E}\left[\left\|U_{N, k}\right\|^{2} 1_{\left\{\left\|U_{N, k}\right\| \geq \varepsilon\right\}}\right] & =\exp \left\{2 T\left\|\nabla_{x} F(\cdot, x(\cdot))\right\|_{\infty}\right\} \Delta^{N} \sum_{k: 0<t_{k}^{N} \leq t} \mathbb{E}\left[\left\|m_{k}^{N}\right\|^{2} 1_{\left.\left\{\left\|m_{k}^{N}\right\| \geq \exp \left(-T\left\|\nabla_{x} F(\cdot, x(\cdot))\right\|_{\infty}\right) \varepsilon\left(\Delta^{N}\right)^{-\frac{1}{2}}\right\}\right]}\right] \\
& \leq c^{2} T \mathbb{E}\left[\sup _{t \in[0, T]}\|\tilde{F}(t, x(t))-F(t, x(t))\|^{2} 1_{\left\{\sup _{t \in[0, T]}\|\tilde{F}(t, x(t))-F(t, x(t))\| \geq c^{-1} \varepsilon\left(\Delta^{N}\right)^{-\frac{1}{2}}\right\}}\right]
\end{aligned}
$$

where $c:=\exp \left(T\left\|\nabla_{x} F(\cdot, x(\cdot))\right\|_{\infty}\right)$. An application of Lebesgue's dominated convergence theorem yields that (4.5) converges to zero as $N$ converges to infinity. We obtain for the third sum in equation (4.3)

$$
\sum_{k: 0<t_{k}^{N} \leq t} \mathbb{E}\left[\left\langle\alpha, Y_{N, k}\right\rangle^{2} 1_{\left\{\left|\left\langle\alpha, Y_{N, k}\right\rangle\right| \geq \varepsilon\right\}}\right] \leq\|\alpha\|^{2} \sum_{k: 0<t_{k}^{N} \leq t} \sum_{i=1}^{d} \mathbb{E}\left[\left|Y_{N, k}^{(i)}\right|^{4}\right]^{\frac{1}{2}} \mathbb{P}\left(\left|\left\langle\alpha, Y_{N, k}\right\rangle\right| \geq \varepsilon\right)^{\frac{1}{2}}
$$

Since, the $d$ elements of the vector $Y_{N, k}$ are normally distributed, we achieve with the notation $\Sigma^{N, k}$ for the covariance matrix $\operatorname{Cov}\left(U_{N, k} U_{N, k}^{\prime}\right)$ that $\sum_{i=1}^{d} \mathbb{E}\left[\left|Y_{N, k}^{(i)}\right|^{4}\right]^{\frac{1}{2}}=\sum_{i=1}^{d} \sqrt{3} \Sigma_{i, i}^{N, k}=\sqrt{3} \mathbb{E}\left[\left\|U_{N, k}\right\|^{2}\right] \leq$ const $\Delta^{N}$, with the help of equation (4.4). In addition, since $\left\langle\alpha, Y_{N, k}\right\rangle \sim \mathcal{N}\left(0,\left\langle\alpha, \Sigma^{N, k} \alpha\right\rangle\right)$, we get

$$
\mathbb{P}\left(\left|\left\langle\alpha, Y_{N, k}\right\rangle\right| \geq \varepsilon\right)^{\frac{1}{2}} \leq \sqrt{2} \Phi\left(-\varepsilon\left\langle\alpha, \Sigma^{N, k} \alpha\right\rangle^{-\frac{1}{2}}\right)^{\frac{1}{2}} \leq \exp \left(-\varepsilon^{2} / 4\|\alpha\|^{-2}\left\|\Sigma^{N, k}\right\|^{-1}\right),
$$

with the help of a bound given in Chiani et al. [2003]. And $\left\|\Sigma^{N, k}\right\| \leq \mathbb{E}\left[\left\|U_{N, k}\right\|^{2}\right] \leq$ const $\Delta^{N}$ from equation (4.4). This proves that (4.6) converges to zero as $N$ tends to infinity. Consequently, $\varphi_{U_{N}}(\alpha)-\varphi_{Y_{N}}(\alpha) \rightarrow 0$, for any $\alpha \in \mathbb{R}^{d}$.

Clearly $Y_{N} \sim \mathcal{N}\left(0, \Sigma^{N}\right)$, where $\Sigma^{N}=\operatorname{Cov}\left(U_{N}, U_{N}\right)$ and can be written as

$$
\begin{aligned}
\Sigma^{N} & =\sum_{k: 0<t_{k}^{N} \leq t}\left(\prod_{j: t_{k}^{N}<t_{j}^{N} \leq t}\left(I+\Delta_{j}^{N} \nabla_{x} F\left(t_{j-1}^{N}, x_{j-1}^{N}\right)\right)\right) \mathbb{E}\left[m_{k}^{N}\left(m_{k}^{N}\right)^{\prime}\right]\left(\prod_{j: t_{k}^{N}<t_{j}^{N} \leq t}\left(I+\Delta_{j}^{N} \nabla_{x} F\left(t_{j-1}^{N}, x_{j-1}^{N}\right)\right)\right)^{\prime}\left(t_{k}^{N}-t_{k-1}^{N}\right) \\
& =\sum_{k: 0<t_{k}^{N} \leq t} P^{N}\left(t_{k}^{N}, t\right) \mathbb{E}\left[\left(\tilde{F}\left(t_{k}^{N}, x_{k}^{N}\right)-F\left(t_{k}^{N}, x_{k}^{N}\right)\right) \cdot\left(\tilde{F}\left(t_{k}^{N}, x_{k}^{N}\right)-F\left(t_{k}^{N}, x_{k}^{N}\right)\right)^{\prime}\right] P^{N}\left(t_{k}^{N}, t\right)^{\prime}\left(t_{k}^{N}-t_{k-1}^{N}\right), \quad(4.7)
\end{aligned}
$$

with the notation $P^{N}(s, t)=\prod_{j: s<t_{j}^{N} \leq t}\left(I+\Delta_{j}^{N} \nabla_{x} F\left(t_{j-1}^{N}, x_{j-1}^{N}\right)\right)$. Due to the continuity of $t \mapsto \nabla_{x} F(t, x(t))$, we get with the help of Lemma A.3 that $P^{N}$ converges uniformly on $[0, T]$ to a continuous matrix valued function $P$. An application of Lebesgue's dominated convergence theorem yields that the map which is defined from the factor in the middle of (4.7) as

$$
\Gamma:[0, T] \rightarrow \mathbb{R}^{d \times d}: \quad t \mapsto \mathbb{E}\left[(\tilde{F}(t, x(t))-(t, x(t))) \cdot(\tilde{F}(t, x(t))-(t, x(t)))^{\prime}\right]
$$

is continuous, thus, $\Sigma^{N}$ converges uniformly on $[0, T]$ to $\int_{0}^{t} P(s, t) \Gamma(s) P(s, t)^{\prime} \mathrm{d} s$.

All in all, $Y_{N}$ converges to $\mathcal{N}\left(0, \int_{0}^{t} P(s, t) \Gamma(s) P(s, t)^{\prime} \mathrm{d} s\right)$ in law. It remains to prove that the summed error terms in (4.1) converge to zero in probability. We start with the first error term. Note that due to the independence, we have for all $j \neq k$ that $\mathbb{E}\left[\left\langle P^{N}\left(t_{j}^{N}, t\right) R_{1}^{N, j}, P^{N}\left(t_{k}^{N}, t\right) R_{1}^{N, k}\right\rangle\right]=0$. Hence, we obtain

$$
\begin{aligned}
& \mathbb{E}\left[\left\|\left(\Delta^{N}\right)^{-\frac{1}{2}} \sum_{k: 0<t_{k}^{N} \leq t} \prod_{j: t_{k}^{N}<t_{j}^{N} \leq t}\left(I+\Delta_{j}^{N} \nabla_{x} F\left(t_{j}^{N}, x_{j}^{N}\right)\right) R_{1}^{N, k}\right\|^{2}\right] \\
& \leq \Delta^{N} \exp \left(2 T\left\|\nabla_{x} F(\cdot, x(\cdot))\right\|_{\infty}\right) \sum_{k=1}^{2^{N}} \mathbb{E}\left[\left\|\left(\tilde{F}_{k}^{N}\left(t_{k}^{N}, \hat{x}_{k}^{N}\right)-\tilde{F}_{k}^{N}\left(t_{k}^{N}, x_{k}^{N}\right)\right)-\left(F_{k}^{N}\left(t_{k}^{N}, \hat{x}_{k}^{N}\right)-F_{k}^{N}\left(t_{k}^{N}, x_{k}^{N}\right)\right)\right\|^{2}\right]
\end{aligned}
$$




$$
\leq \text { const } \Delta^{N} \sum_{k=1}^{2^{N}} \mathbb{E}\left[\sup _{t \in[0, T]}\left\|\hat{x}^{N}(t)-x(t)\right\|^{2}\right] \rightarrow 0 \text { as } N \rightarrow \infty .
$$

By (4.8) the summed first error terms converge to zero in probability. The sum involving the error terms $R_{3}^{N, k}$ is deterministic and converges to zero: We have $\left(\Delta^{N}\right)^{-\frac{1}{2}} \sum_{k: 0<t_{k}^{N} \leq t} \int_{t_{k-1}^{N}}^{t_{k}^{N}}\left\|F\left(t_{k-1}^{N}, x_{k-1}^{N}\right)-F(s, x(s))\right\| \mathrm{d} s \leq$ const $\left(\Delta^{N}\right)^{\frac{1}{2}}$. Finally, consider the sum involving the second error terms $R_{2}^{N, k}$ :

$$
\sum_{k: 0<t_{k}^{N} \leq t} \prod_{j: t_{k}^{N}<t_{j}^{N} \leq t}\left(I+\Delta^{N} \nabla_{x} F\left(t_{j-1}^{N}, x_{j-1}^{N}\right)\right)\left(\Delta^{N}\right)^{\frac{1}{2}}\left\{\left(F\left(t_{k-1}^{N}, \hat{x}_{k-1}^{N}\right)-F\left(t_{k-1}^{N}, x_{k-1}^{N}\right)\right)-\nabla_{x} F\left(t_{k-1}^{N}, x_{k-1}^{N}\right)\left(\hat{x}_{k-1}^{N}-x_{k-1}^{N}\right)\right\} .
$$

We apply the mean value theorem to each component $F_{i}$ of $F$ and get for suitable $\xi_{i}^{N, k-1}$ between $\hat{x}_{k-1}^{N}$ and $x_{k-1}^{N}$ for the norm of equation (4.9) the bound

$$
\begin{aligned}
& \leq\left(\Delta^{N}\right)^{\frac{1}{2}} \exp \left(T\left\|\nabla_{x} F(\cdot, x(\cdot))\right\|_{\infty}\right) \sup _{t \in[0, T]}\left\|\hat{x}^{N}(t)-x(t)\right\| \sum_{0<t_{k}^{N} \leq t} \sqrt{\sum_{i=1}^{d}\left\|\nabla_{x} F_{i}\left(t_{k-1}^{N}, \xi_{i}^{N, k-1}\right)-\nabla_{x} F_{i}\left(t_{k-1}^{N}, x_{k-1}^{N}\right)\right\|^{2}} \\
& \leq\left(\Delta^{N}\right)^{\frac{1}{2}} \exp \left(T\left\|\nabla_{x} F(\cdot, x(\cdot))\right\|_{\infty}\right) \sup _{t \in[0, T]}\left\|\hat{x}^{N}(t)-x(t)\right\| \sum_{0<t_{k}^{N} \leq t} \sum_{i=1}^{d}\left\|\nabla_{x} F_{i}\left(t_{k-1}^{N}, \xi_{i}^{N, k-1}\right)-\nabla_{x} F_{i}\left(t_{k-1}^{N}, x_{k-1}^{N}\right)\right\|
\end{aligned}
$$

Next, define the sets $A^{\rho}:=\left\{y \in \mathbb{R}^{d}:\|y-x(t)\| \leq \rho\right.$ for some $\left.t \in[0, T]\right\}$. Then the functions $\nabla_{x} F_{i}$ are uniformly continuous on $[0, T] \times A^{1}$. Hence, for every $\varepsilon>0$ there is a $\delta>0$ such that for all $(t, x),(s, y) \in[0, T] \times A^{1}$ with $\|(t, x)-(s, y)\|<\delta$, we have $\max _{i=1, \ldots, d}\left\|\nabla_{x} F_{i}(t, x)-\nabla_{x} F_{i}(s, y)\right\|<\varepsilon$. Furthermore, due to the Lipschitzcontinuity of $F$ all gradients $\nabla_{x} F_{i}$ are bounded. Consequently, we obtain for the terms in the sum of equation (4.10) that

$$
\left\|\nabla_{x} F_{i}\left(t_{k-1}^{N}, \xi_{i}^{N, k-1}\right)-\nabla_{x} F_{i}\left(t_{k-1}^{N}, x_{k-1}^{N}\right)\right\| \leq K_{4} 1_{\left\{\sup \left\{\left\|\hat{x}^{N}(t)-x(t)\right\|: t \in[0, T]\right\}>\delta\right\}}+\varepsilon 1_{\left\{\sup \left\{\left\|\hat{x}^{N}(t)-x(t)\right\|: t \in[0, T]\right\} \leq \delta\right\}}
$$

for a suitable constant $0<K_{4}<\infty$. This proves that the expectation of (4.10) can be made arbitrarily small depending on the choice of $\varepsilon>0$ as $N$ converges to infinity. Thus, the summed third error converges to zero in distribution. Hence, the overall error converges in distribution to the constant zero. Theorem 2.7 of Van Der Vaart [1998] states that for random variables $X_{n}$ and $Y_{n}$ such that $X_{n} \Rightarrow X$ and $Y_{n} \Rightarrow c$ where $c$ is a constant, we have $\left(X_{n}, Y_{n}\right) \Rightarrow(X, c)$. This yields the desired asymptotic normality.

\section{Appendix A. Deferred Proofs and Background Material}

Lemma A.1 (Discrete Gronwall Inequality). Let $\left\{f_{k}: k \in \mathbb{N}\right\},\left\{g_{k}: k \in \mathbb{N}\right\},\left\{y_{k}: k \in \mathbb{N}\right\}$ be positive sequences in $\mathbb{R}_{\geq 0}$ which fulfill $y_{n} \leq f_{n}+\sum_{k=0}^{n-1} g_{k} y_{k}$ for every $n \in \mathbb{N}$. Then, we have $y_{n} \leq f_{n}+\sum_{k=0}^{n-1} f_{k} g_{k} \prod_{j=k+1}^{n-1}\left(1+g_{j}\right) \leq$ $f_{n}+\sum_{k=0}^{n-1} f_{k} g_{k} \exp \left(\sum_{j=k+1}^{n-1} g_{j}\right)$ for each $n \in \mathbb{N}$. The first inequality is actually sharp.

Lemma A.2 (Estimates for characteristic functions). Let $X$ be a d-dimensional real random variable on $(\Omega, \mathcal{A}, \mathbb{P})$ and let $\mathcal{G} \subseteq \mathcal{A}$ be a sub- $\sigma$-algebra of $\mathcal{A}$. Define $\mu=\mathbb{E}[X \mid \mathcal{G}]$ and $\Sigma:=\mathbb{E}\left[X X^{\prime} \mid \mathcal{G}\right]$. Then for the conditional characteristic function of $X$ w.r.t. $\mathcal{G},\left.\varphi_{X}\right|_{\mathcal{G}}$, it holds that for each $t \in \mathbb{R}^{d}$

$$
\left.\varphi_{X}\right|_{\mathcal{G}}(t)=\mathbb{E}\left[e^{i\langle t, X\rangle} \mid \mathcal{G}\right]=\left(1-\frac{1}{2}\langle t, \Sigma t\rangle\right) e^{i\langle t, \mu\rangle}+r(t),
$$

where the remainder can be bounded as follows

$$
|r(t)| \leq 2\|t\|^{2} \mathbb{E}\left[\|X\|^{2} 1_{\{\|t\|\|X\|>\varepsilon / 2\}} \mid \mathcal{G}\right]+\varepsilon\|t\|^{2} \mathbb{E}\left[\|X\|^{2} 1_{\{\|t \mid\| X \| \leq \varepsilon\}} \mid \mathcal{G}\right] .
$$

Proof. We can decompose the conditional characteristic function in a real and an imaginary function

$$
\varphi_{X \mid \mathcal{G}}(t)=\mathbb{E}[\cos \langle t, X\rangle \mid \mathcal{G}]+i \mathbb{E}[\sin \langle t, X\rangle \mid \mathcal{G}]
$$

from which we can compute the gradients and the Hessian matrices. We get for the gradient of the sin- and cos-term

$$
t \mapsto-\mathbb{E}[\sin \langle t, X\rangle \cdot X \mid \mathcal{G}] \text { and } t \mapsto \mathbb{E}[\cos \langle t, X\rangle \cdot X \mid \mathcal{G}]
$$

The Hessian matrices are given by

$$
t \mapsto-\mathbb{E}\left[X \cdot \cos \langle t, X\rangle \cdot X^{\prime}\right] \text { and by } t \mapsto-\mathbb{E}\left[X \cdot \sin \langle t, X\rangle \cdot X^{\prime}\right] .
$$


In the first place let $\mathbb{E}[X \mid \mathcal{G}]=0$ a.s. $[\mathbb{P}]$, then

$$
\begin{aligned}
& \mathbb{E}[\cos \langle t, X\rangle \mid \mathcal{G}]=1-\frac{1}{2} \cdot t^{\prime} \cdot \mathbb{E}\left[X \cdot X^{\prime} \mid \mathcal{G}\right] \cdot t-\frac{1}{2} \cdot t^{\prime} \cdot \mathbb{E}\left[X \cdot\left(\cos \left\langle\theta_{1} t, X\right\rangle-1\right) \cdot X^{\prime} \mid \mathcal{G}\right] \cdot t, \\
& \mathbb{E}[\sin \langle t, X\rangle \mid \mathcal{G}]=-\frac{1}{2} \cdot t^{\prime} \cdot \mathbb{E}\left[X \cdot \sin \left\langle\theta_{2} t, X\right\rangle \cdot X^{\prime} \mid \mathcal{G}\right] \cdot t,
\end{aligned}
$$

for suitable $\theta_{1}, \theta_{2} \in[0,1]$ by the mean value theorem. Hence, $\varphi_{X \mid \mathcal{G}}(t)=1-\frac{1}{2}\langle t, \Sigma t\rangle+r(t)$, where

$$
\begin{aligned}
|r(t)|= & \frac{1}{2}\left|\left\langle t,\left\{\mathbb{E}\left[X \cdot\left(\cos \left\langle\theta_{1} t, X\right\rangle-1\right) \cdot X^{\prime} \mid \mathcal{G}\right]+i \mathbb{E}\left[X \cdot \sin \left\langle\theta_{2} t, X\right\rangle \cdot X^{\prime} \mid \mathcal{G}\right]\right\} t\right\rangle\right| \\
\leq & \frac{1}{2} \sup _{\theta_{1} \in[0,1]}\left|\left\langle t, \mathbb{E}\left[X \cdot\left(\exp \left(i\left\langle\theta_{1} t, X\right\rangle\right)-1\right) \cdot X^{\prime} \mid \mathcal{G}\right] t\right\rangle\right| \\
& +\frac{1}{2} \sup _{\theta_{1}, \theta_{2} \in[0,1]}\left|\left\langle t, \mathbb{E}\left[X \cdot\left(\sin \left\langle\theta_{2} t, X\right\rangle-\sin \left\langle\theta_{1} t, X\right\rangle\right) \cdot X^{\prime} \mid \mathcal{G}\right] t\right\rangle\right|
\end{aligned}
$$

Next, we make use of the estimate $\left|1-e^{i \alpha}\right| \leq \min \{|\alpha|, 2\}$ real $\alpha$ for the first term. For the second term, we use that the real sinus function is Lipschitz continuous with Lipschitz-constant 1, i.e. $|\sin x-\sin y| \leq|x-y|$. Hence, we have the following two estimates,

$$
\begin{aligned}
\left|\left\langle t, \mathbb{E}\left[X \cdot\left(\exp \left(i\left\langle\theta_{1} t, X\right\rangle\right)-1\right) \cdot X^{\prime} \mid \mathcal{G}\right] t\right\rangle\right| & \leq\|t\|^{2} \mathbb{E}\left[\|X\|^{2}\left|\exp \left(i\left\langle\theta_{1} t, X\right\rangle\right)-1\right| \mid \mathcal{G}\right] \\
& \leq 2\|t\|^{2} \mathbb{E}\left[\|X\|^{2} 1_{\{\|t\|\|\| X \|>\varepsilon\}} \mid \mathcal{G}\right]+\varepsilon\|t\|^{2} \mathbb{E}\left[\|X\|^{2} 1_{\{\mid\|t\|\|X\| \leq \varepsilon\}} \mid \mathcal{G}\right] .
\end{aligned}
$$

And,

$$
\begin{aligned}
\left|\left\langle t, \mathbb{E}\left[X \cdot\left(\sin \left\langle\theta_{2} t, X\right\rangle-\sin \left\langle\theta_{1} t, X\right\rangle\right) \cdot X^{\prime} \mid \mathcal{G}\right] t\right\rangle\right| \\
\leq 2\|t\|^{2} \mathbb{E}\left[\|X\|^{2} 1_{\{\|t\|\|\| X \|>\varepsilon / 2\}} \mid \mathcal{G}\right]+\varepsilon\|t\|^{2} \mathbb{E}\left[\|X\|^{2} 1_{\{\|t\|\|\| X \| \leq \varepsilon / 2\}} \mid \mathcal{G}\right] .
\end{aligned}
$$

Combining these estimates, the remainder can be bounded as claimed. For general $\mu$, we find that $\varphi_{X \mid \mathcal{G}}(t)=$ $\varphi_{X-\mu}(t) e^{i\langle t, \mu\rangle}$, hence, we can apply the above analysis once again. This finishes the proof.

Lemma A.3. Let $\pi^{N}, N \in \mathbb{N}_{+}$, be the sequence of dyadic partitions of $[0, T], T>0$, such that $0=\tau_{0}^{N}<\tau_{1}^{N}<$ $\ldots<\tau_{2^{N}}^{N}=T$. Let $\chi$ be a continuous matrix valued mapping from $[0, T]$ to $\mathbb{R}^{d \times d}$. Let $\|\cdot\|$ be a submultiplicative matrix norm on $\mathbb{R}^{d \times d}$. Then there is a continuous map

$$
P:[0, T]^{2} \rightarrow \mathbb{R}^{d \times d} \text { such that } \sup _{\substack{s, t \in[0, T] \\ s \leq t}}\left\|\prod_{s<t_{i}^{N} \leq t}\left(I+\Delta_{i}^{N} \chi\left(t_{i-1}^{N}\right)\right)-P(s, t)\right\| \rightarrow 0 \text { as } N \rightarrow \infty .
$$

Proof. For $0 \leq s \leq t \leq T$ write $P^{N}(s, t):=\prod_{s<t_{i}^{N} \leq t}\left(I+\Delta_{i}^{N} \chi\left(t_{i-1}^{N}\right)\right)$. We show that the $\left(P^{N}\right)_{N \in \mathbb{N}_{+}}$are Cauchy w.r.t. $\|\cdot\|_{\infty}$ on $[0, T]$. Fix some $0 \leq s \leq t \leq T$. Let $M \geq N$. Then for a $k \leq 2^{N}$, we have $P^{N}(s, t)=D_{0} \cdot D_{1} \cdot \ldots \cdot D_{k}$, where each $D_{i}=I+\Delta_{i}^{N} \chi\left(t_{i-1}^{N}\right)$ for some $t_{i}^{N} \in(s, t]$ and $D_{0}$ is determined by the unique $t_{l}^{N}$ which fulfills $t_{l-1}^{N} \leq s<t_{l}^{N} \leq t$. Since $\pi^{N} \subseteq \pi^{M}$, we can choose for $i=1, \ldots, k$ the factors

$F_{i}=\left(I+\Delta_{u}^{M} \chi\left(t_{u-1}^{M}\right)\right) \cdot \ldots \cdot\left(I+\Delta_{u+v}^{M} \chi\left(t_{u+v-1}^{M}\right)\right)$ such that for $i \neq l:\left(t_{i-1}^{N}, t_{i}^{N}\right]=\left(t_{u-1}^{M}, t_{u}^{M}\right] \cup \ldots \cup\left(t_{u+v-1}^{M}, t_{u+v}^{M}\right]$ for $v=2^{M-N}-1$. Furthermore, there is a factor $F_{0}$ given by

$$
F_{0}=\left(I+\Delta_{l}^{M} \chi\left(t_{l-1}^{M}\right)\right) \cdot \ldots \cdot\left(I+\Delta_{l+\tilde{v}}^{M} \chi\left(t_{l+\tilde{v}-1}^{M}\right)\right)
$$

for the unique $t_{l}^{M}$ which fulfills $t_{l-1}^{M} \leq s<t_{l}^{M} \leq t$ and $\tilde{v} \leq 2^{M-N}$. Then $P^{M}(s, t)=F_{0} \cdot F_{1} \cdot \ldots \cdot F_{k} \cdot$ Res, where the residual factors of $P^{M}(s, t)$ are collected in Res. Hence, we can write

$$
\begin{aligned}
P^{M}(s, t)-P^{N}(s, t)= & F_{0} \cdot F_{1} \cdot \ldots \cdot F_{k} \cdot \operatorname{Res}-D_{0} \cdot D_{1} \cdot \ldots \cdot D_{k} \\
= & \left(F_{0}-I\right) \cdot F_{1} \cdot \ldots \cdot F_{k} \cdot \operatorname{Res}+F_{1} \cdot \ldots \cdot F_{k} \cdot(\operatorname{Res}-I) \\
& +\left\{F_{1} \cdot \ldots \cdot F_{k}-D_{1} \cdot \ldots \cdot D_{k}\right\}-\left(D_{0}-I\right) \cdot D_{1} \cdot \ldots \cdot D_{k} .
\end{aligned}
$$

Firstly, we have $\|\operatorname{Res}\| \leq \exp \left(\Delta^{N}\|\chi\|_{\infty}\right)$, as well as, $\max \left\{\left\|F_{1} \cdot \ldots \cdot F_{k}\right\|,\left\|D_{1} \cdot \ldots \cdot D_{k}\right\|\right\} \leq \exp \left(T\|\chi\|_{\infty}\right)$ and secondly,

$$
\max \left\{\left\|F_{0}-I\right\|,\left\|D_{0}-I\right\|,\|\operatorname{Res}-I\|\right\} \leq \Delta^{N}\|\chi\|_{\infty} \exp \left(\Delta^{N}\|\chi\|_{\infty}\right) .
$$


Thirdly, we can write the main term as $F_{1} \cdot \ldots \cdot F_{k}-D_{1} \cdot \ldots \cdot D_{k}=\sum_{j=1}^{k} \prod_{i=1}^{j} F_{i} \prod_{i=j+1}^{k} D_{i}-\sum_{j=0}^{k-1} \prod_{i=1}^{j} F_{i} \prod_{i=j+1}^{k} D_{i}$, which implies for the norm of this term

$$
\left\|F_{1} \cdot \ldots \cdot F_{k}-D_{1} \cdot \ldots \cdot D_{k}\right\| \leq \sum_{j=1}^{k} \max _{1 \leq i \leq k}\left\|F_{i}\right\|^{j-1}\left\|F_{j}-D_{j}\right\| \max _{1 \leq i \leq k}\left\|D_{i}\right\|^{k-j} .
$$

The factors of each summand in (A.2) can be estimated as follows, we have for the first and the third factor

$$
\left\|D_{i}\right\| \leq 1+\Delta^{N}\|\chi\|_{\infty} \leq \exp \left(\Delta^{N}\|\chi\|_{\infty}\right) \text { as well as }\left\|F_{i}\right\| \leq\left(1+\Delta^{M}\|\chi\|_{\infty}\right)^{2^{M-N}} \leq \exp \left(\Delta^{N}\|\chi\|_{\infty}\right) .
$$

For the factor in the middle, we use the definition of the modulus of continuity: Define for $\delta>0$ the function $w(\delta, \chi):=\sup \{\|\chi(s)-\chi(t)\|:|s-t| \leq \delta\}$. Then

$$
\left\|F_{j}-D_{j}\right\| \leq \Delta^{N} w\left(\Delta^{N}, \chi\right)+\sum_{j=2}^{2^{M-N}}\left(\begin{array}{c}
2^{M-N} \\
j
\end{array}\right)\left(\Delta^{M}\|\chi\|_{\infty}\right)^{j} \leq \Delta^{N} w\left(\Delta^{N}, \chi\right)+\left(\Delta^{N}\right)^{2}\|\chi\|_{\infty}^{2} \exp \left(\Delta^{N}\|\chi\|_{\infty}\right) .
$$

Eventually, we combine these estimates to find that A.1) can be bounded over all $s$ and $t$ by $c\left(\Delta^{N}+w\left(\Delta^{N}, \chi\right)\right)$, for a suitable constant $0<c<\infty$ which does not depend on $N$. Since $\chi$ is uniformly continuous on $[0, T]$, we have $\lim _{N \rightarrow \infty} w\left(\Delta^{N}, \chi\right)=0$. This proves the Cauchy property and consequently the uniform convergence of the sequence $\left(P^{N}\right)_{N \in \mathbb{N}_{+}}$to a limit function $P$. It remains to prove the continuity of this $P$. We have for all $(s, t),\left(s_{0}, t_{0}\right)$ in $[0, T]^{2}$ that

$$
\begin{aligned}
\left\|P(s, t)-P\left(s_{0}, t_{0}\right)\right\| \leq & \left\|P(s, t)-P^{N}(s, t)\right\|+\left\|P^{N}(s, t)-P^{N}\left(s_{0}, t\right)\right\| \\
& +\left\|P^{N}\left(s_{0}, t\right)-P^{N}\left(s_{0}, t_{0}\right)\right\|+\left\|P^{N}\left(s_{0}, t_{0}\right)-P\left(s_{0}, t_{0}\right)\right\| .
\end{aligned}
$$

And we can compute, that for $0 \leq a<b<c \leq T$, we have

$$
\left\|\prod_{a<i_{i}^{N} \leq b}\left(I+\Delta^{N} \chi\left(t_{i}^{N}\right)\right)-\prod_{a<t_{i}^{N} \leq c}\left(I+\Delta^{N} \chi\left(t_{i}^{N}\right)\right)\right\| \leq\|\chi\|_{\infty} \exp \left(T\|\chi\|_{\infty}\right) \exp \left(\left(c-b+\Delta^{N}\right)\|\chi\|_{\infty}\right)\left((c-b)+\Delta^{N}\right)
$$

where the terms involving the $\Delta^{N}$ stem from the fact that $P^{N}$ is discontinuous at the partitioning points. All in all, the remaining terms in equation $(\mathrm{A} .3 \mathrm{~B}$ ) can be bounded with

$$
\max \left\{\left\|P^{N}(s, t)-P^{N}\left(s_{0}, t\right)\right\|,\left\|P^{N}\left(s_{0}, t\right)-P^{N}\left(s_{0}, t_{0}\right)\right\|\right\} \leq \operatorname{const}\left(\mid \max \left(\left|s-s_{0}\right|,\left|t-t_{0}\right|\right)+\Delta^{N}\right)
$$

This yields the desired continuity of the limit function $P$.

\section{REFERENCES}

Marco Chiani, Davide Dardari, and Marvin K Simon. New exponential bounds and approximations for the computation of error probability in fading channels. Wireless Communications, IEEE Transactions on, 2 (4):840-845, 2003.

Raúl Fierro and Soledad Torres. The euler scheme for hilbert space valued stochastic differential equations. Statistics $\mathcal{E}$ probability letters, 51(3):207-213, 2001.

Raul Fierro and Soledad Torres. A stochastic scheme of approximation for ordinary differential equations. Electronic Communications in Probability, 13:1-9, 2007.

A.W. Van Der Vaart. Asymptotic Statistics. Cambridge Series in Statistical and Probabilistic Mathematics, 3. Cambridge University Press, 1998. ISBN 9780521496032.

E-mail address: krebs@mathematik.uni-kl.de

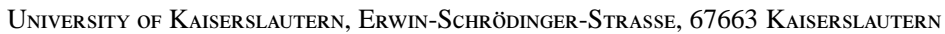

